\title{
Solving Delay Differential Equations by an Accurate Method with Interpolation
}

\author{
Ali Akgül ${ }^{1}$ and Adem Kiliçman ${ }^{2}$ \\ ${ }^{1}$ Department of Mathematics, Education Faculty, Dicle University, 21280 Diyarbakır, Turkey \\ ${ }^{2}$ Department of Mathematics and Institute for Mathematical Research, University Putra Malaysia (UPM), \\ 43400 Serdang, Selangor, Malaysia
}

Correspondence should be addressed to Ali Akgül; aliakgul00727@gmail.com

Received 6 August 2014; Accepted 7 September 2014

Academic Editor: Santanu Saha Ray

Copyright @ 2015 A. Akgül and A. Kiliçman. This is an open access article distributed under the Creative Commons Attribution License, which permits unrestricted use, distribution, and reproduction in any medium, provided the original work is properly cited.

We use the reproducing kernel method (RKM) with interpolation for finding approximate solutions of delay differential equations. Interpolation for delay differential equations has not been used by this method till now. The numerical approximation to the exact solution is computed. The comparison of the results with exact ones is made to confirm the validity and efficiency.

\section{Introduction}

In this paper we consider delay differential equations in the reproducing kernel space:

$$
\begin{gathered}
\frac{1}{s(x)} u^{\prime \prime}(t(x))+\frac{1}{p(x)} u^{\prime}(h(x))+\frac{1}{q(x)} u(m(x))=g(x), \\
u(0)=A, \quad u(1)=B,
\end{gathered}
$$

where $u(x) \in W_{2}^{3}[0,1]$ and $g(x) \in W_{2}^{1}[0,1]$.

The theory of reproducing kernels [1] was used for the first time at the beginning of the 20th century by S. Zaremba in his work on boundary value problems for harmonic and biharmonic functions. In recent years, a lot of attention has been devoted to the study of RKM to investigate various scientific models. The RKM which accurately computes the series solution is of great interest to applied sciences. The method provides the solution in a rapidly convergent series with components that can be elegantly computed. The book [2] provides excellent overviews of the existing reproducing kernel methods for solving various model problems such as integral and integrodifferential equations.
The efficiency of the method was used by many authors to investigate several scientific applications. Geng and Cui [3] applied the RKM to handle the second-order boundary value problems. Wang et al. [4] investigated a class of singular boundary value problems by this method and the obtained results were good. Zhou et al. [5] used the RKM effectively to solve second-order boundary value problems. In [6], the method was used to solve nonlinear infinite-delaydifferential equations. Wang and Chao [7] and Zhou and Cui [8] independently employed the RKM to variable-coefficient partial differential equations. Geng and Cui [9] and Du and Cui [10] researched the approximate solution of the forced Duffing equation with integral boundary conditions by combining the homotopy perturbation method and the RKM. Wu and Li [11] applied iterative reproducing kernel method to obtain the analytical approximate solution of a nonlinear oscillator with discontinuities. Yang et al. [12] used this method for solving the system of the linear Volterra integral equations with variable coefficients. A particular singular integral equation was solved by $\mathrm{Du}$ and Shen [13]. Barbieri and Meo [14] have studied evaluation of the integral terms in reproducing kernel methods. Third-order threepoint boundary value problems were considered by $\mathrm{Wu}$ and $\mathrm{Li}$ [15]. Chen and Chen [16] investigated the exact solution of system of linear operator equations in reproducing kernel 
spaces. Akgül has investigated fractional order boundary value problems by RKM [17]. Inc et al. have solved ordinary and partial differential equations by RKM [18-20].

The paper is organized as follows. Section 2 introduces several reproducing kernel spaces. The associated linear operator is presented in Section 3. Section 4 provides the main results. The exact and approximate solutions of problems and an iterative method are developed in the reproducing kernel space in this section. We have proved that the approximate solutions converge to the exact solutions uniformly. Some numerical experiments are illustrated in Section 5. Some conclusions are given in Section 6.

\section{Preliminaries}

2.1. Reproducing Kernel Spaces. In this section, we define some useful reproducing kernel spaces.

Definition 1 (reproducing kernel function). Let $E \neq \emptyset$. A function $K: E \times E \rightarrow \mathbb{C}$ is called areproducing kernel function of the Hilbert space $H$ if and only if

(a) $K(\cdot, t) \in H$ for all $t \in E$;

(b) $\langle\varphi, K(\cdot, t)\rangle=\varphi(t)$ for all $t \in E$ and all $\varphi \in H$.

The last condition is called "the reproducing property" as the value of the function $\varphi$ at the point $t$ is reproduced by the inner product of $\varphi$ with $K(\cdot, t)$.

Definition 2. We define the space $W_{2}^{3}[0,1]$ by

$$
\begin{array}{r}
W_{2}^{3}[0,1]=\left\{u \in A C[0,1]: u^{\prime}, u^{\prime \prime} \in A C[0,1],\right. \\
\left.u^{(3)} \in L^{2}[0,1], u(0)=u(1)=0\right\} .
\end{array}
$$

The third derivative of $u$ exists almost everywhere since $u^{\prime \prime}$ is absolutely continuous. The inner product and the norm in $W_{2}^{3}[0,1]$ are defined by

$$
\begin{gathered}
\langle u, g\rangle_{W_{2}^{3}}=\sum_{i=0}^{2} u^{(i)}(0) g^{(i)}(0)+\int_{0}^{1} u^{(3)}(x) g^{(3)}(x) \mathrm{d} x, \\
u, g \in W_{2}^{3}[0,1], \\
\|u\|_{W_{2}^{3}}=\sqrt{\langle u, u\rangle_{W_{2}^{3}}}, \quad u \in W_{2}^{3}[0,1] .
\end{gathered}
$$

The space $W_{2}^{3}[0,1]$ is called a reproducing kernel space, as, for each fixed $y \in[0,1]$ and any $u \in W_{2}^{3}[0,1]$, there exists a function $R_{y}$ such that

$$
u(y)=\left\langle u, R_{y}\right\rangle_{W_{2}^{3}} .
$$

Definition 3. We define the space $W_{2}^{1}[0,1]$ by

$$
W_{2}^{1}[0,1]=\left\{u \in A C[0,1]: u^{\prime} \in L^{2}[0,1]\right\} .
$$

The inner product and the norm in $W_{2}^{1}[0,1]$ are defined by

$$
\begin{array}{r}
\langle u, g\rangle_{W_{2}^{1}}=\int_{0}^{1} u(x) g(x)+u^{\prime}(x) g^{\prime}(x) \mathrm{d} x, \\
u, g \in G_{2}^{1}[0,1], \\
\|u\|_{W_{2}^{1}}=\sqrt{\langle u, u\rangle_{W_{2}^{1}}}, \quad u \in W_{2}^{1}[0,1] .
\end{array}
$$

The space $W_{2}^{1}[0,1]$ is a reproducing kernel space, and its reproducing kernel function $T_{x}$ is given by Cui and Lin [2]:

$$
T_{x}(y)=\frac{1}{2 \sinh (1)}[\cosh (x+y-1)+\cosh (|x-y|-1)] .
$$

Lemma 4 (see [21]). The space $W_{2}^{3}[0,1]$ is a reproducing kernel space, and its reproducing kernel function $R_{y}$ is given by

$$
R_{y}(x)= \begin{cases}\sum_{i=1}^{6} c_{i}(y) x^{i-1}, & x \leq y, \\ \sum_{i=1}^{6} d_{i}(y) x^{i-1}, & x>y\end{cases}
$$

where $c_{i}(y)$ and $d_{i}(y)$ coefficients can be found by Maple 16.

\section{Solution Representation in $W_{2}^{3}[0,1]$}

In this section, the solution of (1) is considered in the reproducing kernel space $W_{2}^{3}[0,1]$. On defining the linear operator $L: W_{2}^{3}[0,1] \rightarrow W_{2}^{1}[0,1]$ as

$$
L v(x)=\frac{1}{s(x)} v^{\prime \prime}(t(x))+\frac{1}{p(x)} v^{\prime}(h(x))+\frac{1}{q(x)} v(m(x)),
$$

model problem (1) takes the form

$$
\begin{aligned}
& L v=f(x, v), \quad x \in[0,1], \\
& v(0)=v(1)=0 .
\end{aligned}
$$

In (9), since $v(x)$ is sufficiently smooth, we see that $L$ : $W_{2}^{3}[0,1] \rightarrow W_{2}^{1}[0,1]$ is a bounded linear operator. For convenience, we write $u$ instead of $v$ in (10).

Theorem 5. The linear operator L defined by (9) is a bounded linear operator.

Proof. We only need to prove $\|L u\|_{W_{2}^{1}}^{2} \leq M\|u\|_{W_{2}^{3}}^{2}$, where $M>$ 0 is a positive constant. By (6), we have

$$
\|L u\|_{W_{2}^{1}}^{2}=\langle L u, L u\rangle_{W_{2}^{1}}=\int_{0}^{1}[L u(x)]^{2}+\left[L u^{\prime}(x)\right]^{2} \mathrm{~d} x .
$$

By reproducing property, we have

$$
\begin{gathered}
u(x)=\left\langle u(\cdot), R_{x}(\cdot)\right\rangle_{W_{2}^{3}}, \\
L u(x)=\left\langle u(\cdot), L R_{x}(\cdot)\right\rangle_{W_{2}^{3}},
\end{gathered}
$$


so

$$
|L u(x)| \leq\|u\|_{W_{2}^{3}}\left\|L R_{x}\right\|_{W_{2}^{3}}=M_{1}\|u\|_{W_{2}^{3}},
$$

where $M_{1}>0$ is a positive constant; thus,

$$
\int_{0}^{1}[(L u)(x)]^{2} \mathrm{~d} x \leq M_{1}^{2}\|u\|_{W_{2}^{3}}^{2} .
$$

Since

$$
(L u)^{\prime}(x)=\left\langle u(\cdot),\left(L R_{x}\right)^{\prime}(\cdot)\right\rangle_{W_{2}^{3}},
$$

we have

$$
\left|(L u)^{\prime}(x)\right| \leq\|u\|_{W_{2}^{3}}\left\|\left(L R_{x}\right)^{\prime}\right\|_{W_{2}^{3}}=M_{2}\|u\|_{W_{2}^{3}},
$$

where $M_{2}>0$ is a positive constant, so we have

$$
\begin{gathered}
{\left[(L u)^{\prime}(t)\right]^{2} \leq M_{2}^{2}\|u\|_{W_{2}^{3}}^{2},} \\
\int_{0}^{1}\left[(L u)^{\prime}(x)\right]^{2} \mathrm{~d} x \leq M_{2}^{2}\|u\|_{W_{2}^{3}}^{2},
\end{gathered}
$$

that is

$$
\begin{aligned}
\|L u\|_{W_{2}^{1}}^{2} & \leq \int_{0}^{1}\left([(L u)(x)]^{2}+\left[(L u)^{\prime}(x)\right]^{2}\right) \mathrm{d} x \\
& \leq\left(M_{1}^{2}+M_{2}^{2}\right)\|u\|_{W_{2}^{3}}^{2}=M\|u\|_{W_{2}^{3}}^{2} ;
\end{aligned}
$$

where $M=M_{1}^{2}+M_{2}^{2}>0$ is a positive constant. This completes the proof.

\section{The Structure of the Solution and the Main Results}

From (9), it is clear that $L: W_{2}^{3}[0,1] \rightarrow W_{2}^{1}[0,1]$ is a bounded linear operator. Put $\varphi_{i}(x)=T_{x_{i}}(x)$ and $\psi_{i}(x)=$ $L^{*} \varphi_{i}(x)$, where $L^{*}$ is conjugate operator of $L$. The orthonormal system $\left\{\widehat{\Psi}_{i}(x)\right\}_{i=1}^{\infty}$ of $W_{2}^{3}[0,1]$ can be derived from GramSchmidt orthogonalization process of $\left\{\psi_{i}(x)\right\}_{i=1}^{\infty}$ :

$$
\widehat{\psi}_{i}(x)=\sum_{k=1}^{i} \beta_{i k} \psi_{k}(x), \quad\left(\beta_{i i}>0, i=1,2, \ldots\right) .
$$

Theorem 6. Let $\left\{x_{i}\right\}_{i=1}^{\infty}$ be dense in $[0,1]$ and $\psi_{i}(x)=$ $\left.L_{y} R_{x}(y)\right|_{y=x_{i}}$. Then the sequence $\left\{\psi_{i}(x)\right\}_{i=1}^{\infty}$ is a complete system in $W_{2}^{3}[0,1]$.

Proof. We have

$$
\begin{aligned}
\psi_{i}(x) & =\left(L^{*} \varphi_{i}\right)(x)=\left\langle\left(L^{*} \varphi_{i}\right)(y), R_{x}(y)\right\rangle \\
& =\left\langle\left(\varphi_{i}\right)(y), L y R_{x}(y)\right\rangle=\left.L_{y} R_{x}(y)\right|_{y=x_{i}} .
\end{aligned}
$$

The subscript $y$ by the operator $L$ indicates that the operator $L$ applies to the function of $y$. Clearly, $\psi_{i}(x) \in W_{2}^{3}[0,1]$.
For each fixed $u(x) \in W_{2}^{3}[0,1]$, let $\left\langle u(x), \psi_{i}(x)\right\rangle=0,(i=$ $1,2, \ldots)$, which means that

$$
\left\langle u(x),\left(L^{*} \varphi_{i}\right)(x)\right\rangle=\left\langle L u(\cdot), \varphi_{i}(\cdot)\right\rangle=(L u)\left(x_{i}\right)=0 .
$$

Note that $\left\{x_{i}\right\}_{i=1}^{\infty}$ is dense in $[0,1]$; hence, $(L u)(x)=0$. It follows that $u \equiv 0$ from the existence of $L^{-1}$. So the proof of Theorem 6 is completed.

Theorem 7. If $u(x)$ is the exact solution of (10), then

$$
u(x)=\sum_{i=1}^{\infty} \sum_{k=1}^{i} \beta_{i k} f\left(x_{k}, u_{k}\right) \widehat{\Psi}_{i}(x),
$$

where $\left\{\left(x_{i}\right)\right\}_{i=1}^{\infty}$ is dense in $[0,1]$.

Proof. From (19) and uniqueness of solution of (10), we have

$$
\begin{aligned}
u(x) & =\sum_{i=1}^{\infty}\left\langle u(x), \widehat{\Psi}_{i}(x)\right\rangle_{W_{2}^{3}} \widehat{\Psi}_{i}(x) \\
& =\sum_{i=1}^{\infty} \sum_{k=1}^{i} \beta_{i k}\left\langle u(x), \Psi_{k}(x)\right\rangle_{W_{2}^{3}} \widehat{\Psi}_{i}(x) \\
& =\sum_{i=1}^{\infty} \sum_{k=1}^{i} \beta_{i k}\left\langle u(x), L^{*} \varphi_{k}(x)\right\rangle_{W_{2}^{3}} \widehat{\Psi}_{i}(x) \\
& =\sum_{i=1}^{\infty} \sum_{k=1}^{i} \beta_{i k}\left\langle L u(x), \varphi_{k}(x)\right\rangle_{W_{2}^{1}} \widehat{\Psi}_{i}(x) \\
& =\sum_{i=1}^{\infty} \sum_{k=1}^{i} \beta_{i k}\left\langle f(x, u), T_{x_{k}}\right\rangle_{W_{2}^{1}} \widehat{\Psi}_{i}(x) \\
& =\sum_{i=1}^{\infty} \sum_{k=1}^{i} \beta_{i k} f\left(x_{k}, u_{k}\right) \widehat{\Psi}_{i}(x) .
\end{aligned}
$$

This completes the proof.

Now the approximate solution $u_{n}(x)$ can be obtained from the $n$-term intercept of the exact solution $u$ and

$$
u_{n}(x)=\sum_{i=1}^{n} \sum_{k=1}^{i} \beta_{i k} f\left(x_{k}, u_{k}\right) \widehat{\Psi}_{i}(x) .
$$

Lemma 8 (see [22]). If $\left\|u_{n}-u\right\|_{W_{2}^{3}} \rightarrow 0, x_{n} \rightarrow x,(n \rightarrow$ $\infty)$, and $f(x, u)$ is continuous for $x \in[0,1]$, then

$$
f\left(x_{n}, u_{n-1}\left(x_{n}\right)\right) \longrightarrow f(x, u(x)) \text { as } n \longrightarrow \infty \text {. }
$$

Lemma 9 (see [23]). For any fixed $u_{0}(x) \in W_{2}^{3}[0,1]$, suppose the following conditions are satisfied:

(i)

$$
\begin{gathered}
u_{n}(x)=\sum_{i=1}^{n} A_{i} \widehat{\psi}_{i}(x), \\
A_{i}=\sum_{k=1}^{i} \beta_{i k} f\left(x_{k}, u_{k-1}\left(x_{k}\right)\right) .
\end{gathered}
$$

(ii) $\left\|u_{n}\right\|_{W_{2}^{3}}$ is bounded;

(iii) $\left\{x_{i}\right\}_{i=1}^{\infty}$ is dense in $[0,1]$;

(iv) $f(x, u) \in W_{2}^{1}[0,1]$ for any $u(x) \in W_{2}^{3}[0,1]$. 
TABLE 1: Approximate solutions of Example 12.

\begin{tabular}{ccc}
\hline$x$ & $\begin{array}{c}\text { Approximate solution } \\
(m=20)\end{array}$ & $\begin{array}{c}\text { Approximate solution } \\
(m=40)\end{array}$ \\
\hline 0.1 & -0.14567324629372310303 & -0.14332570351241038744 \\
0.2 & -0.29985556099058424878 & -0.29495827353958461228 \\
0.3 & -0.45709584460083115842 & -0.44946832894209791578 \\
0.4 & -0.61194299763471154735 & -0.60142648858052541270 \\
0.5 & -0.75894592060247313735 & -0.74540337131544229233 \\
0.6 & -0.89265351401436369206 & -0.875969596007423769 \\
0.7 & -1.0076146783806308025 & -0.987695781517044783 \\
\hline
\end{tabular}

Then $u_{n}(x)$ in iterative formula (26) converges to the exact solution of (22) in $W_{2}^{3}[0,1]$ and

$$
u(x)=\sum_{i=1}^{\infty} A_{i} \widehat{\psi}_{i}(x),
$$

where $A_{i}$ is given by (27).

We assume that $\left\{x_{i}\right\}_{i=1}^{\infty}$ is dense in $[0,1]$. Let $u(x)$ be the exact solution of (1) and let $u_{n}(x)$ be the $n$-term approximation solution of (1). We set

$$
\|u\|_{C}=\max _{x \in 0,1]}|u(x)| .
$$

Theorem 10. If $u \in W_{2}^{3}[0,1]$, then

$$
\left\|u_{n}-u\right\|_{W_{2}^{3}} \longrightarrow 0, \quad n \longrightarrow \infty .
$$

Moreover, a sequence $\left\|u_{n}-u\right\|_{W_{2}^{3}}$ is monotonically decreasing in $n$.

Proof. From (22) and (24), it follows that

$$
\left\|u_{n}-u\right\|_{W_{2}^{3}}=\left\|\sum_{i=n+1}^{\infty} \sum_{k=1}^{i} \beta_{i k} f\left(x_{k}, u_{k}\right) \widehat{\Psi}_{i}\right\|_{W_{2}^{3}} .
$$

Thus,

$$
\left\|u_{n}-u\right\|_{W_{2}^{3}} \longrightarrow 0, \quad n \longrightarrow \infty
$$

In addition,

$$
\begin{aligned}
\left\|u_{n}-u\right\|_{W_{2}^{3}}^{2} & =\left\|\sum_{i=n+1}^{\infty} \sum_{k=1}^{i} \beta_{i k} f\left(x_{k}, u_{k}\right) \widehat{\Psi}_{i}\right\|_{W_{2}^{3}}^{2} \\
& =\sum_{i=n+1}^{\infty}\left(\sum_{k=1}^{i} \beta_{i k} f\left(x_{k}, u_{k}\right) \widehat{\Psi}_{i}\right)^{2} .
\end{aligned}
$$

Clearly, $\left\|u_{n}-\mathfrak{u}\right\|_{W_{2}^{3}}$ is monotonically decreasing in $n$.

Remark 11. Let us consider countable dense set $\left\{x_{1}, x_{2}, \ldots\right\} \in$ $[0,1]$ and define

$$
\varphi_{i}=T_{x_{i}}, \quad \Psi_{i}=L^{*} \varphi_{i}, \quad \widehat{\Psi}_{i}=\sum_{k=1}^{1} \beta_{i k} \Psi_{k} .
$$

TABLE 2: Approximate solutions of Example 13.

\begin{tabular}{lcc}
\hline$x$ & $\begin{array}{c}\text { Approximate solution } \\
(m=20)\end{array}$ & $\begin{array}{c}\text { Approximate solution } \\
(m=40)\end{array}$ \\
\hline $1 / 128$ & 1.007699352586986977 & 1.0078430972064479777 \\
$1 / 64$ & 1.015467872441933409 & 1.0157477085866857475 \\
$1 / 32$ & 1.0312145777006698521 & 1.0317781924005388582 \\
$1 / 16$ & 1.0635619468092503034 & 1.0646422184208995853 \\
$1 / 8$ & 1.1317728332594306196 & 1.1336864923985190672 \\
$1 / 4$ & 1.2820670848004034655 & 1.2853973453552029427 \\
$3 / 8$ & 1.4506420021011940437 & 1.455402968114665988 \\
\hline
\end{tabular}

TABLE 3: Approximate solutions of Example 14.

\begin{tabular}{ccc}
\hline$x$ & $\begin{array}{c}\text { Approximate solution } \\
(m=20)\end{array}$ & $\begin{array}{c}\text { Approximate solution } \\
(m=40)\end{array}$ \\
\hline 0.4 & 0.37198086110266406119 & 0.36562543902764212178 \\
0.6 & 0.56420118043724686324 & 0.55433514429791597661 \\
0.7 & 0.66467348067813109719 & 0.65290860208735486704 \\
0.8 & 0.76971776926603696502 & 0.75593299892958661386 \\
0.9 & 0.8808862311855404085 & 0.86493995475233692321 \\
$3 / 8$ & 0.34846550611241626259 & 0.34252746655728479953 \\
$5 / 8$ & 0.58896615156191184948 & 0.57863565938605001693 \\
$7 / 8$ & 0.85242546043516774048 & 0.83703396768442560044 \\
\hline
\end{tabular}

Then $\beta_{i k}$ coefficients can be found by

$$
\begin{aligned}
& \beta_{11}=\frac{1}{\left\|\Psi_{1}\right\|}, \quad \beta_{i i}=\frac{1}{\sqrt{\left\|\Psi_{i}\right\|^{2}}-\sum_{k=1}^{i-1} c_{i k}^{2}}, \\
& \beta_{i j}=\frac{-\sum_{k=j}^{i-1} c_{i k} \beta_{k j}}{\sqrt{\left\|\Psi_{i}\right\|^{2}}-\sum_{k=1}^{i-1} c_{i k}^{2}}, \quad c_{i k}=\left\langle\Psi_{i}, \widehat{\Psi}_{k}\right\rangle .
\end{aligned}
$$

4.1. Interpolation for Reproducing Kernel Method. We used interpolation to find the numerical results by RKM with

$$
\begin{aligned}
u(x)= & \frac{(x-b)(x-c)}{(a-b)(a-c)} u(a) \\
& +\frac{(x-a)(x-c)}{(b-a)(b-c)} u(b)+\frac{(x-a)(x-b)}{(c-b)(c-a)} u(c),
\end{aligned}
$$

where $0.1 \leq a<x<b<c \leq 1$. More details for interpolation can be found in [24].

\section{Numerical Results}

In this section, four numerical examples are provided to show the accuracy of the present method. We used interpolation for Examples 12-14. The RKM does not require discretization of the variables, that is, time and space; it is not effected by computation round-off errors and one is not faced with necessity of large computer memory and time. The accuracy of the RKM for the delay differential equation is controllable and absolute errors are small with present choice of $x$ (see Tables 1-6). The numerical results we obtained justify the advantage of this methodology. 
TABle 4: Numerical results of Example 15.

\begin{tabular}{lccc}
\hline$x$ & Exact solution & Approximate solution $(m=20)$ & Approximate solution $(m=40)$ \\
\hline 0.1 & 0.001 & 0.00090776461553418538803 & 0.00098904754340427120243 \\
0.2 & 0.008 & 0.0079254004789810215967 & 0.0079919839363928962286 \\
0.3 & 0.027 & 0.026940356780732013097 & 0.026994411062098332384 \\
0.4 & 0.064 & 0.063953086850948970952 & 0.063996421220125258843 \\
0.5 & 0.125 & 0.12496400548134896886 & 0.12499809894400479331 \\
0.6 & 0.216 & 0.21597349594471182527 & 0.21599952248650893754 \\
0.7 & 0.343 & 0.34298191656657060634 & 0.343000765213362709 \\
0.8 & 0.512 & 0.51198960694198087943 & 0.51200189692520825458 \\
0.9 & 0.729 & 0.72899689388568731882 & 0.72900298512674395795 \\
1.0 & 1.000 & 1.000 & 1.000 \\
\hline
\end{tabular}

TABLE 5: Absolute error for Example 15.

\begin{tabular}{lcc}
\hline$x$ & Absolute error $(m=20)$ & Absolute error $(m=40)$ \\
\hline 0.1 & $9.223538446581461197 \times 10^{-5}$ & $1.095245659572879757 \times 10^{-5}$ \\
0.2 & $7.45995210189784033 \times 10^{-5}$ & $8.0160636071037714 \times 10^{-6}$ \\
0.3 & $5.9643219267986903 \times 10^{-5}$ & $5.588937901667616 \times 10^{-6}$ \\
0.4 & $4.6913149051029048 \times 10^{-5}$ & $3.578779874741157 \times 10^{-6}$ \\
0.5 & $3.599451865103114 \times 10^{-5}$ & $1.90105599520669 \times 10^{-6}$ \\
0.6 & $2.650405528817473 \times 10^{-5}$ & $4.7751349106246 \times 10^{-7}$ \\
0.7 & $1.808343342939366 \times 10^{-5}$ & $7.65213362709 \times 10^{-7}$ \\
0.8 & $1.039305801912057 \times 10^{-5}$ & $1.89692520825458 \times 10^{-6}$ \\
0.9 & $3.10611431268118 \times 10^{-6}$ & $2.98512674395795 \times 10^{-6}$ \\
1.0 & 0.0 & 0.0 \\
\hline
\end{tabular}

Example 12. Consider the equation

$$
\begin{aligned}
& \frac{1}{p(x)} u^{\prime}(g(x))+\frac{1}{q(x)} u(h(x)) \\
& \quad+\frac{1}{r(x)} u(x)+\frac{1}{s(x)} u^{\prime}(x)=F(x), \\
& u(0)=0=u(1),
\end{aligned}
$$

where

$$
\begin{aligned}
& g(x)=\sqrt{x}, \quad h(x)=\sqrt[3]{x}, \\
& p(x)=x, \quad q(x)=x^{2}, \\
& r(x)=x-1, \quad s(x)=x+1, \quad F(x)=\exp (x) .
\end{aligned}
$$

Thus, if the method described above is applied, then we find Table 1.

Example 13. We take notice of equation

$$
\begin{aligned}
& u^{\prime \prime}(x)=\left(u\left[\frac{x}{(1+2 x)^{2}}\right]\right)^{(1+2 x)^{2}}, \\
& u(0)=1, \quad u(1)=\exp (1)
\end{aligned}
$$

We use transformation

$$
v(x)=u(x)-x(\exp (1)-1)-1
$$

to obtain

$$
\begin{aligned}
& v^{\prime \prime}(x) \\
& \quad=\left(v\left[\frac{x}{(1+2 x)^{2}}+\frac{x}{(1+2 x)^{2}}(\exp (1)-1)+1\right]\right)^{(1+2 x)^{2}} \\
& v(0)=0, \quad v(1)=0 .
\end{aligned}
$$

Thus, if the method described above is applied, then we find Table 2.

Example 14. We regard the following equation:

$$
\begin{aligned}
& u^{\prime \prime}(x)=u\left(x^{2}\right), \\
& u(0)=0, \quad u(1)=1 .
\end{aligned}
$$

We use transformation

$$
v(x)=u(x)-x
$$


TABLE 6: Relative error for Example 15.

\begin{tabular}{lcc}
\hline$x$ & Relative error $(m=20)$ & Relative error $(m=40)$ \\
\hline 0.1 & $9.223538446581461197 \times 10^{-2}$ & $1.095245659572879757 \times 10^{-2}$ \\
0.2 & $9.3249401273723004125 \times 10^{-3}$ & $1.002007950887971425 \times 10^{-3}$ \\
0.3 & $2.209008121036551963 \times 10^{-3}$ & $2.0699770006176355556 \times 10^{-4}$ \\
0.4 & $7.33017953922328875 \times 10^{-4}$ & $5.5918435542830578125 \times 10^{-5}$ \\
0.5 & $2.8795614920824912 \times 10^{-4}$ & $1.520844796165352 \times 10^{-5}$ \\
0.6 & $1.2270395966747560185 \times 10^{-4}$ & $2.2107106067706481481 \times 10^{-6}$ \\
0.7 & $5.2721380260622915452 \times 10^{-5}$ & $2.2309427484227405248 \times 10^{-6}$ \\
0.8 & $2.0298941443594863281 \times 10^{-5}$ & $3.7049320473722265625 \times 10^{-6}$ \\
0.9 & $4.2607878088905075446 \times 10^{-6}$ & $4.0948240657859396433 \times 10^{-6}$ \\
1.0 & 0.0 & 0.0 \\
\hline
\end{tabular}

to obtain

$$
\begin{aligned}
& v^{\prime \prime}(x)=v\left(x^{2}\right)+x^{2}, \\
& v(0)=0, \quad v(1)=0 .
\end{aligned}
$$

Thus, if the method described above is applied, then we find Table 3.

Example 15. We consult equation

$$
\begin{aligned}
& u^{\prime \prime}(x)=u(|x|)+|x|\left(6-x^{2}\right), \\
& u(-1)=1, \quad u(1)=1 .
\end{aligned}
$$

We use transformation

$$
v(x)=u(x)-|x|
$$

to obtain

$$
\begin{aligned}
& v^{\prime \prime}(x)=v(|x|)+|x|\left(7-x^{2}\right), \\
& v(-1)=0, \quad v(1)=0 .
\end{aligned}
$$

The exact solution of (45) is given as

$$
u(x)=|x| x^{2} .
$$

Thus, if the method described above is applied, then we find Tables 4, 5, and 6 .

\section{Conclusion}

In this paper, we introduced an algorithm for finding approximate solutions of delay differential equations with RKM. For illustration purposes, four examples were selected to show the computational accuracy. It may be concluded that the RKM is very powerful and efficient in finding approximate solutions for wide classes of problems. Solutions obtained by the present method are uniformly convergent. As shown in Tables 1-6, results of numerical examples show that the present method is an accurate and reliable analytical method for these problems. The present study has confirmed that the RKM offers significant advantages in terms of its straightforward applicability, its computational effectiveness, and its accuracy to solve the strongly nonlinear equations.

\section{Conflict of Interests}

The authors declare that they do not have any competing interests or conflict of interests.

\section{References}

[1] N. Aronszajn, “Theory of reproducing kernels," Transactions of the American Mathematical Society, vol. 68, pp. 337-404, 1950.

[2] M. Cui and Y. Lin, Nonlinear Numerical Analysis in the Reproducing Kernel Space, Nova Science, New York, NY, USA, 2009.

[3] F. Geng and M. Cui, "Solving a nonlinear system of second order boundary value problems," Journal of Mathematical Analysis and Applications, vol. 327, no. 2, pp. 1167-1181, 2007.

[4] W. Wang, M. Cui, and B. Han, "A new method for solving a class of singular two-point boundary value problems," Applied Mathematics and Computation, vol. 206, no. 2, pp. 721-727, 2008.

[5] Y. Zhou, Y. Lin, and M. Cui, "An efficient computational method for second order boundary value problems of nonlinear differential equations," Applied Mathematics and Computation, vol. 194, no. 2, pp. 354-365, 2007.

[6] X. Lü and M. Cui, "Analytic solutions to a class of nonlinear infinite-delay-differential equations," Journal of Mathematical Analysis and Applications, vol. 343, no. 2, pp. 724-732, 2008.

[7] Y. Wang and L. Chao, "Using reproducing kernel for solving a class of partial differential equation with variable-coefficients," Applied Mathematics and Mechanics, vol. 29, no. 1, pp. 129-137, 2008.

[8] S. Zhou and M. Cui, "Approximate solution for a variablecoefficient semilinear heat equation with nonlocal boundary conditions," International Journal of Computer Mathematics, vol. 86, no. 12, pp. 2248-2258, 2009.

[9] F. Geng and M. Cui, "New method based on the HPM and RKHSM for solving forced Duffing equations with integral boundary conditions," Journal of Computational and Applied Mathematics, vol. 233, no. 2, pp. 165-172, 2009.

[10] J. Du and M. Cui, "Solving the forced Duffing equation with integral boundary conditions in the reproducing kernel space," International Journal of Computer Mathematics, vol. 87, no. 9, pp. 2088-2100, 2010.

[11] B. Wu and X. Li, "Iterative reproducing kernel method for nonlinear oscillator with discontinuity," Applied Mathematics Letters, vol. 23, no. 10, pp. 1301-1304, 2010. 
[12] L.-H. Yang, J.-H. Shen, and Y. Wang, “The reproducing kernel method for solving the system of the linear Volterra integral equations with variable coefficients," Journal of Computational and Applied Mathematics, vol. 236, no. 9, pp. 2398-2405, 2012.

[13] H. Du and J. Shen, "Reproducing kernel method of solving singular integral equation with cosecant kernel," Journal of Mathematical Analysis and Applications, vol. 348, no. 1, pp. 308314, 2008.

[14] E. Barbieri and M. Meo, "A fast object-oriented Matlab implementation of the reproducing kernel particle method," Computational Mechanics, vol. 49, no. 5, pp. 581-602, 2012.

[15] B. Wu and X. Li, "Application of reproducing kernel method to third order three-point boundary value problems," Applied Mathematics and Computation, vol. 217, no. 7, pp. 3425-3428, 2010.

[16] Z. Chen and Z. Chen, "The exact solution of system of linear operator equations in reproducing kernel spaces," Applied Mathematics and Computation, vol. 203, no. 1, pp. 56-61, 2008.

[17] A. Akgül, "A new method for approximate solutions of fractional order boundary value problems," Neural Parallel Scientific Computations, vol. 22, no. 12, 2014.

[18] M. Inc and A. Akgül, "Approximate solutions for MHD squeezing fluid flow by a novel method," Boundary Value Problems, vol. 2014, article 18, 2014.

[19] M. Inc, A. Akgül, and A. Kiliçman, "Explicit solution of telegraph equation based on reproducing kernel method," Journal of Function Spaces and Applications, vol. 2012, Article ID 984682, 23 pages, 2012.

[20] M. Inc, A. Akgül, and A. Kiliçman, "A novel method for solving $\mathrm{KdV}$ equation based on reproducing kernel Hilbert space method," Abstract and Applied Analysis, vol. 2013, Article ID 578942, 11 pages, 2013.

[21] M. Inc and A. Akgül, "The reproducing kernel Hilbert space method for solving Troesch's problem," Journal of the Association of Arab Universities for Basic and Applied Sciences, vol. 14, no. 1, pp. 19-27, 2013.

[22] F. Geng and M. Cui, "Solving singular nonlinear second-order periodic boundary value problems in the reproducing kernel space," Applied Mathematics and Computation, vol. 192, no. 2, pp. 389-398, 2007.

[23] M. Inc, A. Akgül, and F. Geng, "Reproducing kernel Hilbert space method for solving Bratu's problem," Bulletin of the Malaysian Mathematical Sciences Society. In press.

[24] R. P. Agarwal and Y. M. Chow, "Finite-difference methods for boundary value problems of differential equations with deviating arguments," Computers \& Mathematics with Applications, vol. 12, no. 11, pp. 1143-1153, 1986. 


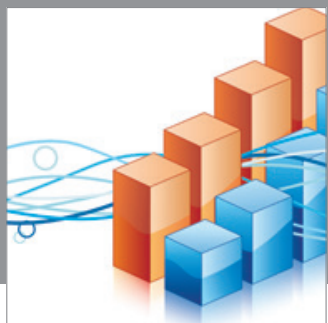

Advances in

Operations Research

mansans

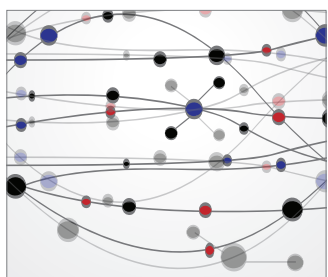

The Scientific World Journal
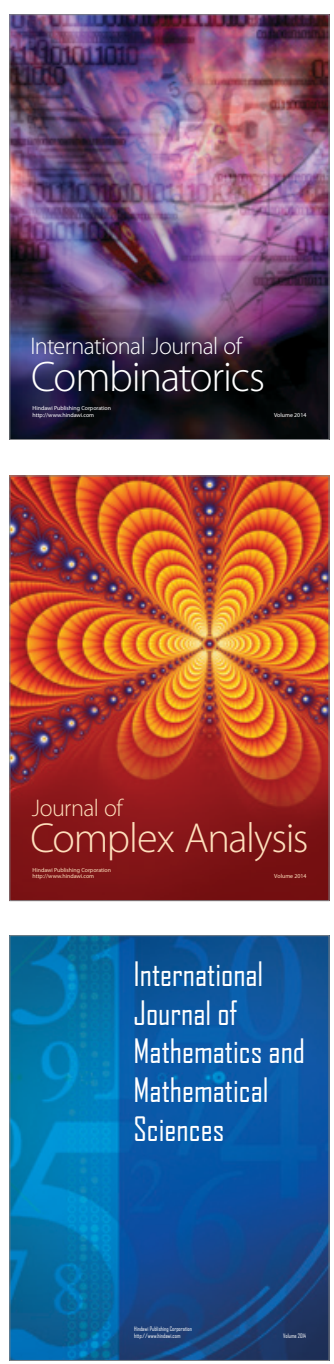
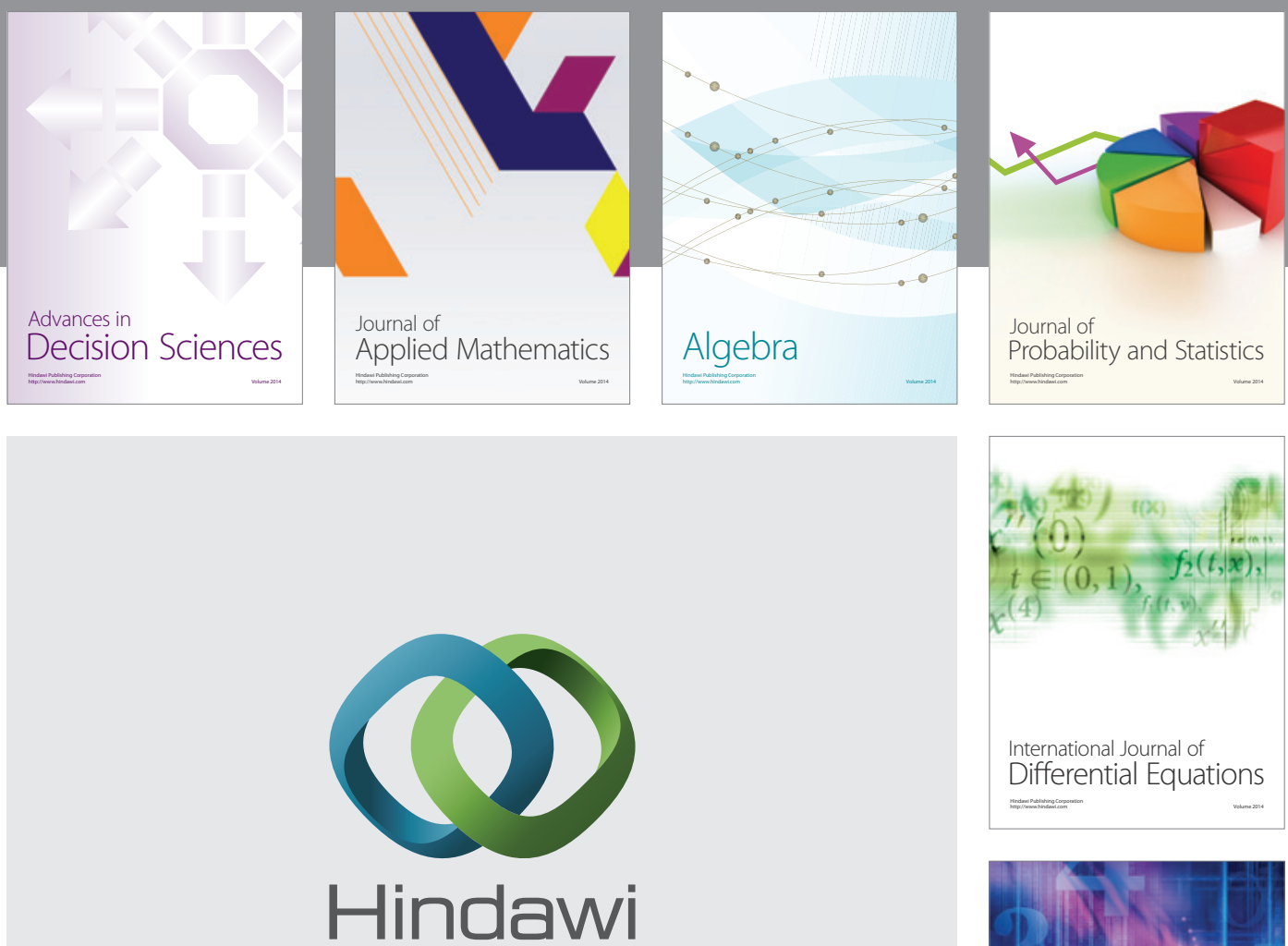

Submit your manuscripts at http://www.hindawi.com
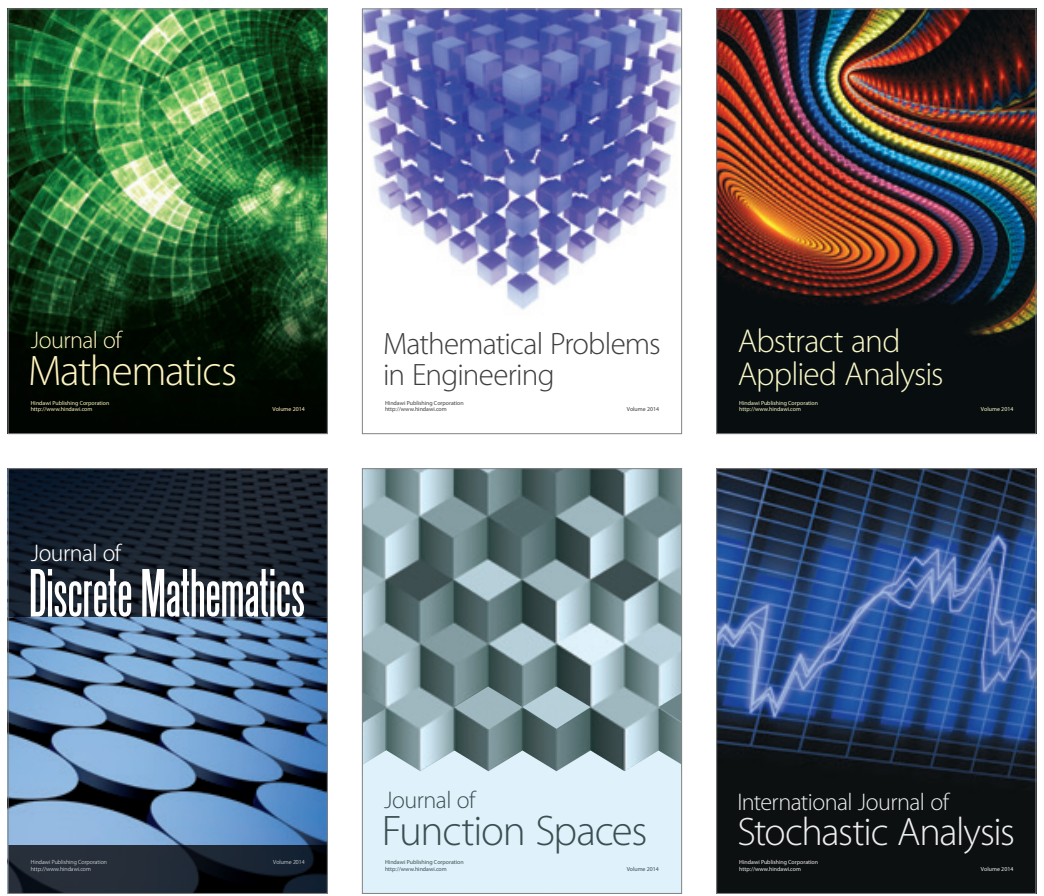

Journal of

Function Spaces

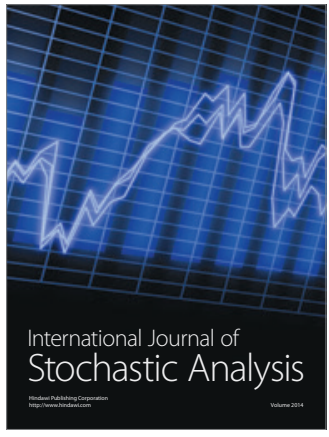

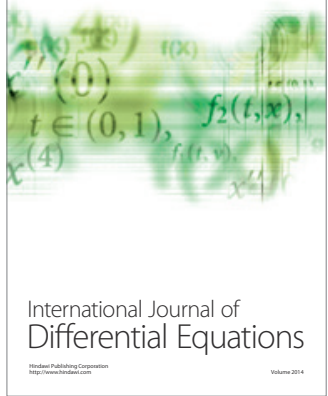
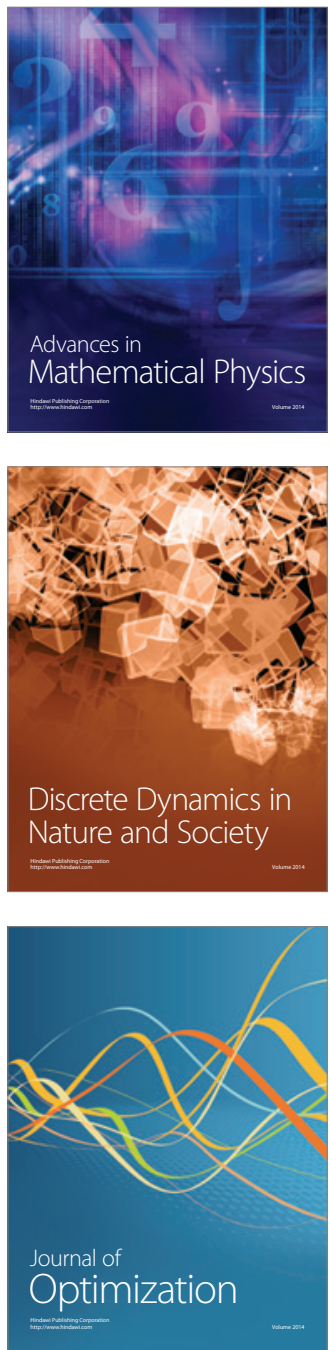